\title{
Analysis of methodological approaches and de- velopment of principles for describing properties characterizing the dynamics of the emission and spreading of toxic components of plants of the fuel and energy complex
}

\author{
Vadim Bespalov, Oxana Gurova*, Marina Volodina, and Lydmila Alekseenko \\ Don State Technical University, 344000 Rostov-on-Don, Russia
}

\begin{abstract}
The article gives a complete description of the pollutants properties and their behavior patterns based on studies considering the peculiarities of a continuous medium, on the one hand, and a set of discrete particles of different sizes, on the other. For that purpose, the authors used the theory of disperse systems, based on the classical concepts of colloidal and physical chemistry. The article presents the methodology developed by the authors to generalize, systemize and describe the properties and behaviour patterns of toxic components of exhaust gases from public and domestic power units, power plants, and exhaust gases from mobile power units for the dynamic conditions for their formation and spreading in the air basin of urban areas. It allows controlling the behavior of toxic components of exhaust gases in order to reduce air pollution and ensure the environmental safety in the builtup areas.
\end{abstract}

\section{Introduction (topicality)}

The dynamics of air pollution is determined by the existence and concentration of various types of pollutants including toxic components of plants of the fuel and energy complex (FEC). Multiyear research has shown [1] that:

- when burning natural gas nitrogen oxide, nitrogen dioxide, carbon monoxide and benz(a)pyrene should be considered as the main toxic components;

- when burning liquid fuel sulfur oxides and ash particles are added to the abovelisted substances;

- when burning solid fuel, in addition to the polluting substances, a significant amount of solid particles consisting of ash particles, unburned solid fuel and soot is emitted.

During a certain period of scientific development, the methodological basis for evaluating the characteristics of toxic components that pollute the air of builtup areas during the operation of FEC plants came down to the study of individual properties and phenomena, and to the determination of the parameters corresponding to them. Thus, the authors of papers [1] investigated the optical properties of aerosol particles, their

\footnotetext{
* Corresponding author: okgurova@yandex.ru
} 
dimensions, particles sedimentation rate, electrization and coagulation of aerosols. The authors of papers [2] presented the principles for evaluating the physicochemical properties of pollutants (in particular industrial dust) based on the determination of the following characteristics: distribution of dust particles, mechanical properties, chemical composition. The papers [3] present the results of a detailed study of the morphological properties of aerosol particles. However, such approaches complicate the complex description of the pollutants behavior in the air, and, therefore, make it difficult to select highly efficient air purification systems from the toxic components of various plants, and especially the plants of the fuel and energy complex significantly contributing to air pollution of urban areas. That is why the task of systematization of parameters characterizing the properties and behavior of pollutants is very topical, since it will allow to carry out a purposeful and consistent evaluation of all dynamics aspects of the formation, emission, distribution and destruction of pollutants.

\section{Research objective}

As a result of research conducted by many authors, the main parameters and properties of toxic components polluting the air environment of builtup areas were studied. It provided means for not only specifying the known classification of aerosols, but also to better understanding the physics and chemistry of their formation and evolution [4-9]. However, it stands to mention a certain chaos in the selection and study of certain properties of toxic components that pollute the air of builtup areas [10].

Moreover, the methodological approaches proposed for studying the properties and behavioral patterns of toxic components can be divided into groups in the following two main directions [12]:

- the first direction involves the analysis of particles of toxic components as discrete material bodies, whose behavior follows the laws of Newtonian mechanics. However, considering the polydispersity of aerosols containing toxic components, this direction accurately describes the behavior of relatively large particles with significant dimensions and masses, but with a large error describes the behavior of small particles that have relatively small dimensions and mass. As a result, it fails to predict the behavior of the polluting aerosol with a reasonable degree of accuracy;

- the second direction involves the analysis of a polluting aerosol, which contains particles of toxic components, as a continuous medium (cloud), whose behavior must follow the laws of the theory of continuous media. However, also considering the polydispersity of aerosols containing toxic components, this direction describes quite accurately the behavior of relatively small particles that are of sufficiently small dimensions and mass, but with a significant error describes the behavior of large particles that are relatively large in dimensions and mass. In this case, too, it fails to predict the behavior of the polluting aerosol with a reasonable degree of accuracy.

Obviously, not to obtain the onesided general picture of the description of the pollutant aerosol behavior, it requires a full specification of the properties and a comprehensive description of the pollutants behavior on the basis of studies considering the features of a continuous medium, on the one hand, and a lot of discrete particles of different dimensions, on the other hand. Such a problem can be solved using the theory of disperse systems [1013], which is based on the classical concepts of colloidal and physical chemistry. 


\section{Theoretical framework}

The basis of the research is the classification characteristics of disperse systems according to the applied theory: by the aggregative state of the dispersed phase and the disperse medium; by the aggregative state of the phase components; by the degree of dispersion; by the intensity of interphase interaction; by the porosity of the dispersed phase; by the shape of particles; by origin.

In accordance with the basic principles of the theory of disperse systems, one may state that, with a certain reserve of free total surface energy WSaer., the studied toxic components, like any disperse system as a whole, display in the air environment certain features of the dynamics of emission and spreading, which is finally characterized by their stability [14]. According to the papers of many researchers [14,15], stability is the combined characteristic that determines the behavior and existence of toxic components as disperse systems, i.e. the parameter of their "viability". Summing up the research of many authors, stability is reasonable to be considered as a characteristic of the degree (speed) of the parameters changing which determine the properties and energy of toxic components over a certain time interval under conditions of external influences. Considering toxic components as disperse systems, it can be said that they are thermodynamically nonequilibrium systems due to their large specific surface area resulting in a tendency to decrease the free total surface energy WSaer with a decrease in the specific surface area Ssp. You must consider that the more stable the disperse system, the slower its parameters change, and vice versa. However, the instability of toxic components as a disperse system can find expression either in the particles coarsening (recrystallization) or, more often, in their coalescence and the formation of aggregates (coagulation). Thus, air purification based on the theory of disperse systems comes to a drastic fall in the stability of toxic components resulting in their destruction, that is, to the irretrievable separation of the dispersed phase and the disperse medium.

Analysis of the known methodological approaches in the two above mentioned directions [10] to the determina-tion and evaluation of the properties of toxic components allows us to conclude that currently poor attention is paid to the systematization of the parameters determining their behavior in the air basin of urban areas. Considering the toxic components of exhaust gases as disperse systems, it can be concluded that a complex, sequential study of their state should be conducted based on an ordered consideration of the parameters characterizing the properties of the dispersed phase and the disperse medium $[14,15]$.

For this purpose, the parameters determining the properties of the disperse phase and disperse medium we referred to the following groups: geometric parameters (GPd.ph., GPd.m.), physicochemical parameters (PCPd.ph., PCPd.m.), optical parameters (OP d.ph., OP d.m.), aerodynamic parameters (ADP d.ph., AD d.m.), hydrodynamic parameters (GDPd.ph., GDPd.m..), thermophysical parameters (TPPd.ph., TPPd.m.), electromagnetic parameters (EMPd.ph, EMPd.m.). The main classification feature of these groups of parameters is the physics of the processes and phenomena observed in polluting aerosols in the form of toxic components $[14,15]$.

As a result of this consideration of the parameters determining the properties of the dispersed phase and the disperse medium of such aerosols, the set of parameters determining the properties of the aerosol (PSaer.) can be generally represented as a functional relationship between groups of parameters of the aerosol phase components $[14,15]$ :

$$
\begin{gathered}
\text { PSaer. }=f 1(P S \text { d.ph }, P S \text { d.m. })=f 1(f(G P d . p h . ., P C P d . p h ., O P \text { d.ph., ADP d.ph., } \\
\text { GDPd.ph, TPPd.ph., EMPd.ph.), } f(G P d . m ., P C P d . m . ., \\
\text { OP d.m., AD d.m, GDPd.m..., TPPd.m., EMPd.m), }
\end{gathered}
$$


where PS ${ }_{\text {d.ph. }}$ - the set of parameters determining the properties of the aerosol dispersed phase; PS d.m. - the set of parameters determining the properties of the aerosol disperse medium.

Such a generalization and systematization of the parameters allows us to complete each group of parameters of the disperse phase and the disperse medium with new characteristics in the development of theoretical bases and to perform a purposeful and sequential estimation of all aspects of the dynamics of formation, emission, spreading and destruction of toxic components $[14,15]$.

The acquisition, distribution and consumption of energy by toxic components as dispersed systems is quantitatively characterized by energy parameters, which are determined by an interconnected complex of groups of properties parameters of the dispersed phase and the disperse medium. Depending on which groups of parameters the properties of toxic components are characterized, their energy state will be described by the corresponding energy parameters. Considering that the processes of formation, emission, spreading and destruction of toxic components occur in real conditions for a long period and can be considered unlimited in time, the energy aerosol parameters should be considered relating to time unit and expressed in terms of specific energy in Watts.

The following main energy parameters were determined from the selected groups by the appropriate combination of the parameters of the properties of the dispersed phase and the disperse medium of toxic components [14,15]: $W_{k i n}$, which characterizes the energy of motion (kinetic energy); $W_{\text {opt. }}$, which characterizes the quantum energy (the energy of optical interaction); $W_{\text {adh. }}$, which characterizes the energy of adhesive interaction (coagulation energy); $W_{h d}$, which characterizes the energy of hydrodynamic interaction (energy of wetting, spreading, absorption, evaporation, condensation); $W_{t h}$, characterizing thermal energy; $W_{e m}$, characterizing electromagnetic energy. The studied types of energy all together determine the free total surface energy $W_{\text {Saer }}$ (activation energy) of toxic components, which can be described by the following implicit dependence [14-15]:

$$
\begin{aligned}
& W_{\text {Saer }}=f_{14}\left(W_{S \text { d.ph. }}, W_{S \text { d.m. }}\right)=f_{14}\left(f_{14.1}\left(W_{\text {kin. }} \text { (d.ph. }\right),\right. \\
& \left.W_{\text {opt.(d.ph.), }} W_{\text {adh.(d.ph.), }} W_{\text {hd(d.ph.), }} W_{\text {th.(d.ph.), }} W_{\text {em(d.ph.) }}\right) \text {, } \\
& f_{14.2} \text { ( } W_{\text {kin.(d.m.) }}, W_{\text {opt. (d.m.). }}, W_{\text {adh.(d.m.) }}, W_{\text {hd(d.m.). }} \text {, } \\
& \left.W_{\text {th. (d.m.). }}, W_{\text {em(d.m.). }}\right) \text {. }
\end{aligned}
$$

Thus, the above mentioned free energy of toxic components is functionally dependent on the different energy parameters of the dispersed phase and the disperse medium. It provides the possibility of redistribution of certain types of energy reflecting the behavior of toxic components that pollute the air basin of urban areas. Such redistribution allows controlling the state of toxic components, which, in turn, will greatly facilitate the selection of highly efficient air purification systems from toxic components of the plants of the fuel and energy complex.

\section{Practical relevance}

The conducted stage of the study of toxic components as dynamic disperse systems allowed to develop the principles for describing the properties and their behavior patterns including the following main steps:

- step 1: identification of the type of toxic component;

- step 2: determination of the list and actual values of the $P S_{a e r}$. properties parameters of the considered type of toxic component as an aerosol, including determination of the properties parameters of its dispersed phase $P S_{\mathrm{d} \text {.ph. }}$ and the disperse medium of $P S_{\mathrm{d} . \mathrm{m} .}$;

- step 3:referring the properties parameters of $P S_{\mathrm{d} . p h}$. and $P S_{\mathrm{d} . \mathrm{m}}$. of toxic component to 
one of the groups identified by us, the main classification feature of which is the physics of the processes and phenomena observed in aerosols;

- step 4: research, the list formation and determination of the values of the main energy parameters determining the dynamics of the aerosol: $W_{\text {kin }}\left(\right.$ d.ph.), $W_{\text {opt (d.ph.) }}, W_{\text {adh }}\left(\right.$ d.ph.), $W_{h d}$ (d.ph.), $W_{\text {th.(d.ph.) }}, W_{\text {em(d.ph.) }}, W_{\text {kin (d.m.), }}, W_{\text {opt (d.m.) }}, W_{\text {adh (d.m.), }}, W_{\text {hd (d.m.) }}, W_{\text {th.(d.m.) }}, W_{\text {em(d.m.). }}$, which characterize the acquisition, distribution and consumption of energy by toxic components, and, ultimately, their free total surface energy $W_{\text {S.aer. }}$ and are determined by the interconnected complex of parameters groups of the $P S_{\text {aer }}$ properties;

- step 5: determination of the direction (technique) of stability changing $U_{\text {aer }}$ of toxic component for the purpose of its destruction as a dynamic disperse system on the basis of certain values of its basic energy parameters. This technique of stability changing $U_{\text {aer }}$. should be the basis for the selection of highly efficient air purification systems from toxic components of fuel and energy companies that make a significant contribution to the pollution of the air basin of urban areas.

\section{Conclusion}

As a result of the conducted research, we developed principles for generalizing, systematizing and describing the properties and behavior patterns of toxic components of exhaust gases. It allows us to solve the problem of controlling the behavior patterns of toxic components of exhaust gases from public and domestic power units, power plants, as well as exhaust gases from mobile power units under the dynamic conditions of the air basin of the urban area in order to reduce air pollution and ensure the environmental safety in builtup areas.

\section{References}

1. N.V. Yudina, E.P. Lysova, L.Z. Ganicheva, A collection of papers on the materials of the International Scientific and Practical Conference, Tambov, 7, 161 (2014)

2. H. Green, V. Leyn, Aerosols - dust, smoke and fog (Leningrad, 1972)

3. P.A. Kouzov, L.Ya. Scriabina, Methods for determining the physicochemical properties of industrial dusts (Leningrad, 1983)

4. P. Raist, Aerosols - an introduction to the theory (Moscow, 1987)

5. L.S. Ivlev, Chemical composition and structure of atmospheric aerosols (Leningrad, 1982)

6. L.S. Ivlev, Optical properties of atmospheric aerosols (Leningrad, 1986)

7. L.S. Ivlev, Physics of atmospheric aerosol systems (St. Petersburg, 1999)

8. K.Ya. Kondratiev, N.I. Moskalenko, D.V. Pozdnyakov, Atmospheric aerosol (Leningrad, 1983)

9. N.A. Fuks, Mechanics of aerosols (Moscow, 1955)

10. L.A. Shvartsman, M.A. Menkovsky, Physical and Colloid Chemistry (Moscow, 1981)

11. D.A. Friedrichsberg, Course of colloid chemistry (Leningrad, 1983)

12. P.A. Rebinder, Colloid chemistry (Moscow, 1978)

13. V.I. Bespalov, Proceedings of the North Caucasian Scientific Center of Higher Education. Natural Sciences, 37 (1995)

14. E.P. Lysova, A.P. Pirozhnikova, Construction-2013, 122 (2013)

15. E.P. Lysova, L.A. Lysutina, All-Russian scientific and practical conference devoted to the Year of Environmental Protection and the 65th anniversary of the Ufa State Oil Technical University, Ufa, 98 (2013) 